\title{
Acute Myeloid Leukaemia Presenting as Gaze Palsy
}

\author{
Evripidis Sykakis $^{a}$ Showrob N. Patwary
}

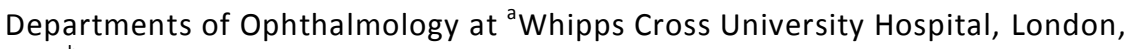
and ${ }^{b}$ Wolverhampton Eye Infirmary, Wolverhampton, UK

\section{Key Words}

Acute myeloid leukaemia $\cdot$ Gaze palsy $\cdot$ Chloroma

\begin{abstract}
The most frequent initial ocular manifestation of acute myeloid leukaemia (AML) is retinal involvement. Here, we report an unusual case of AML associated with a pontine chloroma presenting with gaze palsy as the initial symptom. A 77-year-old Caucasian man presented to the Eye Casualty complaining of a one-day history of blurred vision. On examination, his face was turned to the left, both eyes were fixed in dextroversion and the patient demonstrated left gaze palsy associated with left motor neurone VII palsy. Baseline blood investigations revealed leucocytosis with $60 \%$ circulating myeloblasts. A bone marrow biopsy confirmed the diagnosis of myelomonocytic leukaemia. A CT scan showed a well-circumscribed lesion in the dorsal pons, most likely representing a chloroma. Chloromas or myeloblastomas related to AML are localised extramedullary tumours composed of leukaemic myeloid cells. Chloromas may be present at the time of the initial diagnosis of leukaemia or may precede the diagnosis by 1 month to 2 years; however, their occurrence in the central nervous system is rare, comprising $1-6 \%$ of all chloromas. This case illustrates the many different ways that AML can manifest itself in the eyes, and ophthalmologists should be aware of the great variety of presenting symptoms in undiagnosed AML.
\end{abstract}

\section{Introduction}

Acute myeloid leukaemia (AML) can manifest itself in the eyes in many ways, the most common generally being retinal involvement [1]. The most common intracranial complication in patients with AML is intracranial haemorrhage [2, 3]. AML presenting as gaze palsy is extremely rare, and in the current case it was related to a chloroma (tumour composed of myeloid cells) in the dorsal pons. 


\section{Case Report}

A 77-year-old Caucasian man presented to the Eye Casualty complaining of a one-day history of blurred vision. He also complained that over the last 2 weeks he had been feeling lethargic and experiencing episodes of dizziness lasting for a few minutes. He had no previous ocular or medical problems and was on no regular medications. On clinical examination, the patient's vision was 6/9 in both eyes. His pupils were equal and normally reactive to light and there was no relative afferent pupillary defect. His face was turned to the left and both eyes were fixed in dextroversion, with normal dextroelevation and dextrodepression. However, the patient demonstrated complete left lateral conjugate gaze palsy for voluntary saccadic, pursuit and oculovestibular movements. He also had right-over-left hypertropia and horizontal jerk nystagmus with fast phase to the right, which was increased on right gaze (supplementary videos). Oculocephalic manipulation did not improve the defective ocular response and examination of the rest of the cranial nerves revealed mild left lower motor neurone VII palsy affecting the whole left side of the face. Anterior segments were white and quiet, and intraocular pressure was within normal limits; dilated fundoscopy revealed a few small haemorrhages throughout both retinae, including an inferior juxtapapillary haemorrhage in the left eye (fig. 1). The appearance of the optic discs was normal. Baseline blood investigations including full blood count, erythrocyte sedimentation rate, levels of C-reactive protein, urea, electrolytes and blood glucose, lipid profile, clotting, and immunoglobulin screen were performed. The results revealed mild anaemia, moderate leucocytosis with $60 \%$ circulating myeloblasts, and mild thrombocytopenia; while the erythrocyte sedimentation rate was slightly elevated for the patient's age, levels of C-reactive protein were normal. A bone marrow biopsy confirmed the diagnosis of myelomonocytic leukaemia (M3, French-American-British classification). A CT scan showed a well-circumscribed lesion in the dorsal pons, associated with a small calcification, representing a chloroma (fig. 2).

Following the diagnosis of AML, the patient declined the standard chemotherapy regime for his condition, taking into consideration the small difference in the prognosis with or without treatment, for his age group [4]. Follow-up was arranged with our orthoptist service, where homonymously leftbased prisms of 12 dioptres were used to improve head posture.

\section{Discussion}

AML presenting as gaze palsy is extremely rare and, to our knowledge, has not been previously reported in the literature. Hsu et al. [5] reported a case of AML presenting as one-and-a-half syndrome as a result of acute brainstem infarction. In our case, the symptoms and signs could specifically locate the lesion to the dorsal pons, in close proximity to the left paramedian pontine reticular formation (PPRF) and the left VII nerve fasciculus. The PPRF lies within the medial portion of the pontine tegmentum and is located ventral to the VI nucleus. The motor VII nucleus lies in the pontine tegmentum dorsal to the superior olive. The axons of the neurones in the motor VII nucleus pass medially, rostrally and dorsally to course over the VI nucleus to form the facial colliculus in the floor of the fourth ventricle and continue as the VII nerve fasciculus, which at this point is in close proximity to the ipsilateral PPRF. This is the specific anatomic area affected by the chloroma in our patient, which was in agreement with the CT imaging findings. In this case, the lesion in the left PPRF was preventing voluntary saccadic and pursuit eye movements to the left of midline. Both eyes were fixed to dextroversion because of the unopposed normal circuitry of the respective right horizontal saccades. Additionally, the lesion in the fibres of the motor VII nerve resulted in the observed facial weakness ipsilateral to the lesion.

Chloromas or myeloblastomas related to acute myeloid leukaemia are localised extramedullary tumours composed of leukaemic myeloid cells. The first case of chloroma was reported in 1811 in a patient with proptosis, but it was not until 1892 
when its association with myelogenic leukaemia was established. Chloromas may be present at the time of the initial diagnosis of leukaemia or may precede the diagnosis by 1 month to 2 years. These tumours can present almost anywhere in the body, but their occurrence in the central nervous system is rare, comprising $1-6 \%$ of all chloromas $[6$, 7].

This case illustrates the many different ways that AML can manifest itself in the eyes, and ophthalmologists should be aware of the great variety of presenting symptoms in undiagnosed AML.

\section{Disclosure Statement}

The authors declare that they have no conflicts of interest.

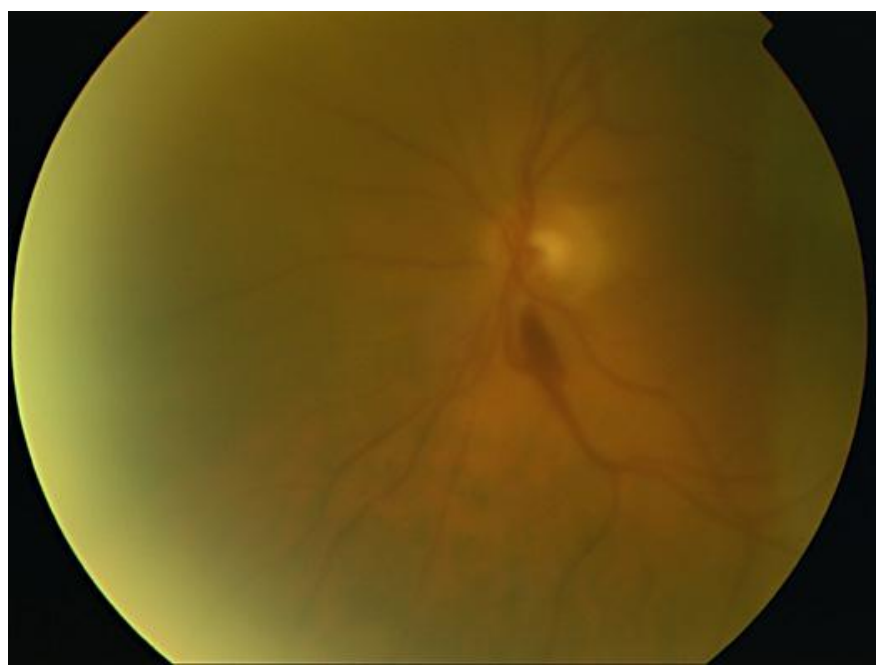

Fig. 1. Fundus photograph of the left eye (quality compromised because of gaze palsy). 


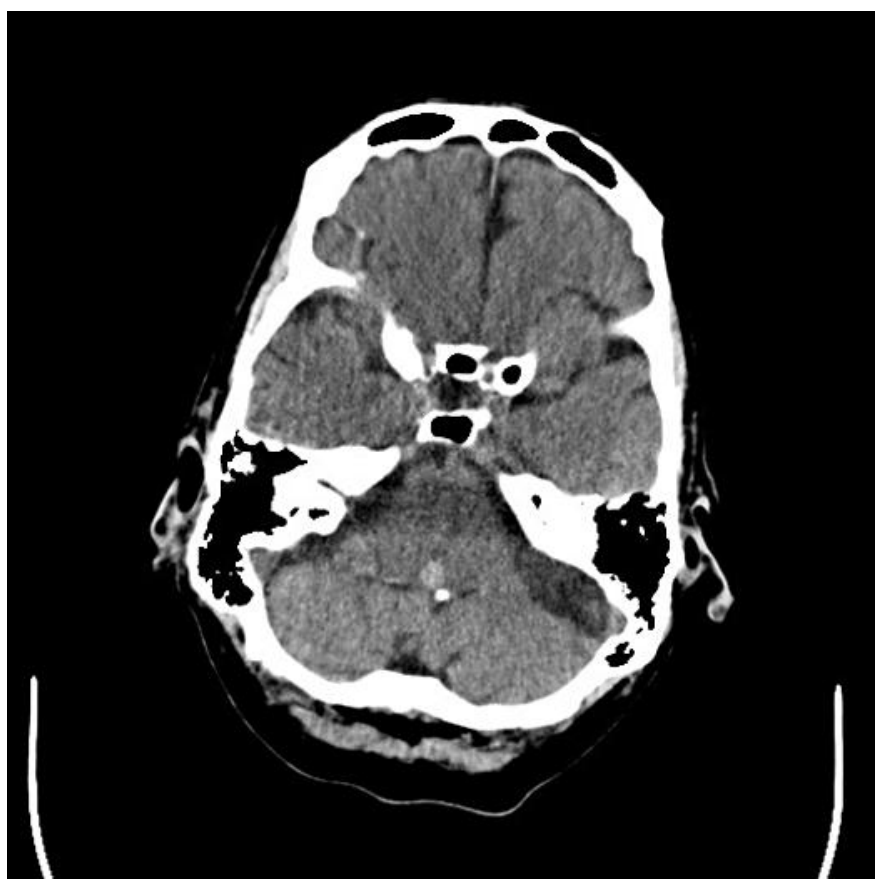

Fig. 2. Axial cut of contrast-enhanced CT scan of the head showing a lesion in the dorsal pons, associated with a small calcification.

\section{References}

1 Schachat AP, et al: Ophthalmic manifestations of leukemia. Arch Ophthalmol 1989;107:697-700.

- $2 \mathrm{Kim} \mathrm{H}$, et al: Analysis of fatal intracranial hemorrhage in 792 acute leukemia patients. Haematologica 2004;89:622-624.

-3 Creutzig U, et al: Early deaths due to hemorrhage and leukostasis in childhood acute myelogen ous leukemia. Associations with hyperleukocytosis and acute monocytic leukemia. Cancer 1987;60:30713079 .

4 Menzin J, Lang K, Earle CC, Kerney D, Mallick R: The outcomes and costs of acute myeloid leukemia among the elderly. Arch Intern Med 2002;162:1597-1603.

5 Hsu WH, Tsai WC, Chu SJ, Tsao YT: Acute myeloid leukemia presenting as one-and-a-half syndrome. Am J Emerg Med 2008;26:513.e1-e2.

-6 Simpson TA, Anderson ML, Garcia JH, Barton JC: Myeloblastoma of the brain. Acta Neuropathol 1989;78:444-447.

7 Imrie KR, et al: Isolated chloroma: the effect of early antileukemic therapy. Ann Intern Med 1995;123:351-353. 